\title{
Assessment of Efficacy of Homoeopathic Remedies for the Treatment of Uncomplicated Malaria ('A Case Study in Effiduase, Sekyere East District of Ashanti Region' Ghana)
}

\author{
Article by Eric Gyamfi ${ }^{1}$, John Awuni Abugire ${ }^{2}$, Takyi Clifford ${ }^{3}$, Abigail Adusei ${ }^{4}$ \\ ${ }^{1}$ Withrow University College, Ghana \\ ${ }^{2,3,4}$ College of Alternative Medicine, Ghana \\ E-mail: gyamfieric2008@yahoo.com
}

\begin{abstract}
Globally, Malaria Is Noted To Be Among That Important Diseases Related To The Environment. However, Problem Related To Malarial Still Remains Very Enamours With Little Improvement. Much Is Interventions Are Needed To Be Done Particularly In Most Africa And Its Sub-Regions. Owing To A Widespread Resistance to Previously Known Anti-Malarial Chemotherapies, the Revision of Antimalarial Drug Policies by Most Sub-Saharan African Countries Is Essential

It Is Of This Concern That This Controlled Clinical Trial Study Was Conducted To Assess The Efficacy, Safety And Tolerability Of Single And Combination Antimalarial Homoeopathic Remedies For The Treatment Of Uncomplicated Malaria In The Sekeyer East District Of Ashanti Region, Ghana.

The Study Documented The Following At The End Of The Trial. No Statistically Significance Difference Was Observed Between The Two Treatment Groups Of The Trial. A Percentage Efficacy Of 95.8\% And 95\% Was Observed For Treatment with Single Remedy and Combination Remedy Respectively. Again Participants On Control Group Showed No Improvement On Malaria Status At The End Of The Study. This Proved A Significant Efficacy of Homoeopathic Anti-Malaria Remedies.

With Reference To These Finding, The Study Recommend The Following As Well; Homoeopathy Remedies Be Advocated For Nationwide Usage As It Has About 90\% To 95\% Success Rate For The Treatment Of Uncomplicated Malaria With No Or Minimal Side Effect, The Research Should Be Reproduced In A Different Geographical Location Involving Large Samples Of Participants To Confirm The Validity Of The Present Findings And To Enhance Generalization.
\end{abstract}

Keywords: Un complication malaria, Homoeopathic remedies, Clinical trials, Sekeyere East District.

\section{Background of the Study}

Among the most importance environmental related disease, malaria is considered one among the numerous. The problem of malarial is much felt in sub-Saharan Africa regions where Plasmodium falciparum, is believed to be causing large numbers of infections resulting in uncontrollable illness and dead each year as noted by Greenwood et al., 2005.

In recent times, there have an advances resulting in optimism regarding the control of malaria, which eventually have led to a decrease in incidence of all malarial recorded episodes in most of Africa. (Kleinschmidt et al., 2006).

However, the problem still not fully attended to and thus an improved malaria control strategies and interventions are required for successful management of the case. Prompt administration of highly effective therapies to those ill with malaria is said to be among the important component of malaria control programme. WHO current recommendations emphasis on the treatment of uncomplicated malaria in Africa with artemisinin-based combination therapy (ACT) owing to a widespread increasing level resistance to older therapies ever reported.

Owing to an increasing availability of highly effective and efficient mode of treatment including ACTs, factors other than efficacy, including safety and tolerability, ease of administration and cost will be measures to influence selection of the optimal regimen. Evaluations on the basis of safety and tolerability of new regimens comparatively will become increasingly important for informing therapeutic decisions and policy.

Several decades ago, the approach for malaria treatment was the single course treatment with drug, which facilitated the increased resistance to most drugs by malaria parasites. This has played a 
DOI: 10.21522/TIJPH.2013.05.04.Art006

ISSN: $2520-3134$

significant role in contributing to numerous avoidable illnesses and deaths attributable to malaria. However, although parasites can easily adapt to resist one drug, adapting to combinations of two or three potent and efficacious drugs is less likely to occur.

On the basis of this we studied the comparative efficacy of single and combination homoeopathic antimalarial remedies on clinically malarial ill-health patients in the Sekyere East district of Ashanti region to ascertain the effectiveness of homeopathic remedies for the therapeutic action on malaria.

\section{Rationale of the study}

With increasing worldwide dead attributable to malaria, there is the need to implement a rapid but most effective intervention. This call for multiple approaches to estimated death of malarial control and prevention. An estimated death of 1 to 3 million occurs each year worldwide, believed to be caused my malaria, particularly young children in sub-Saharan Africa. This is precipitated by many factors including but not limited to increased drug resistance, poor malaria prevention programs and policies coupled with and a frequent inability of patients to pay for treatment. This ultimately indicates that, effective therapy is desperately needed in most African countries than any other parts of the world.

However, because of differences in drug resistance between and within regions, a drug combination will not necessarily work everywhere. With increasing competition for healthcare resources, development partners and policymakers are more likely to subsidize the most cost-effective treatments options for malaria prevention and control.

The researchers aimed to provide policymakers with information on how different homoeopathic anti-malaria remedies are efficacious in the treatment of uncomplicated malaria to ensure the best selection of remedies for treatment of diseases, with least cost options.

\section{Research questions}

The study attempts answering the following research question

1. What are the constitutions of the respondents?

2. How is the initial malaria status of the respondents

3. What is the final malaria status of the respondents after the intervention?

\section{Research hypothesis}

The study investigated the hypothesis below as the main consideration of the study.

- Homeopathic single remedy has the same therapeutic effect in the treatment of uncomplicated malaria compared to combination remedy.

\section{Main objective of the study}

The study was principally meant to assess and compare the efficacy, safety and tolerability of single and combination antimalarial homoeopathic remedies for the treatment of uncomplicated malaria

\section{Specific objectives}

Specifically, the intended to:

- Compare the effectiveness of homeopathic single remedy and combination remedies for the treatment of uncomplicated malaria.

- Generate baseline document for further researches

\section{Materials and methods}

\section{Study area}

The study was conducted in Effiduasi in the Sekyere East district of Ashanti Region, Ghana. The study principally took place on filed with the laboratory analysis at the Effiduasi Government hospital. The hospital has a daily average attendance of about 300 patients. It is the main source of health care centre for the neighbouring environment, thus act as a referral point. The district occupies an area of $714 \mathrm{~km}^{2}$ in the forest belt. Geographically, the study area is homogeneous. All distances between villages are $<15 \mathrm{~km}$ with altitude levels between 300 and 500 metres above the sea level. Temperature varies between $20.4^{\circ} \mathrm{C}$ and $33.5^{\circ} \mathrm{C}$ while monthly rainfall ranges from $15 \mathrm{~mm}$ (January) 
to $214 \mathrm{~mm}$ (June) with similar fluctuation in all villages. The area is holoendemic for P. falciparum malaria with perennial transmission and seasonal peaks high transmission from May to October.

\section{Study type and design}

The study was an interventional clinical trial carried out to assess and compare the efficacy, safety, and tolerability of single and combination antimalarial homoeopathic remedies for treatment of uncomplicated malaria among selected residents of Effiduasi in Ashanti region of Ghana. One of the trial group evaluated treatment of single homoeopathic remedy on uncomplicated malaria, the other trial group evaluated treatment of combination homoeopathic remedies for uncomplicated malaria whilst control group received no active homoeopathic remedy.

\section{Ethical consideration}

The study protocol was reviewed and approved by the College of Alternative Medicine Research Committee. An informed verbal consent was obtained from all participants before they were included in the study. The probability and magnitude of harm or discomfort anticipated by the study was not greater than those encountered in daily life or during the performance of routine physical activities. The study therefore presented no or minimal risk, for which participants were made aware from the beginning of the study. Any other effects of the study were also made known to participant. Only consented individual were enrolled in the study.

\section{Pre-inclusion exercise}

Before participants were enrolled in the study, a total of 100 individuals were screened for presence of malaria parasite in their blood samples, accompanied with clinical history taken of each individual. Only 44 were eligible to be enrolled in the study, thus the study enrolled a total of 44 participants in the study.

\section{Eligibility criteria}

The following were the basis on which respondents were enrolled in the study whereas the other side or the reverse of under listed was considered the exclusion criteria for participation.

\section{Inclusion criteria}

- $\quad$ Aged 18 and above

- $\quad$ Both sex

- Absence of severe health condition

- A slide-confirmed infection with P. falciparum only (i.e. no mixed infections)

- $\quad$ Measured axillary temperature $\geq 37.5^{\circ} \mathrm{C}$

- $\quad$ Ability to attend stipulated follow-up visits

- Informed consent provided by individual

- Absence of history of hypersensitivity reactions to homeopathic drugs

- $\quad$ Resident in the study area

\section{Intervention}

The following were the homoeopathic interventions for the study

- Remedy : Hepar Sulphericum

- Remedy: Aconite

- Remedy: Belladonna

- Remedy: Gelsemium

- Remedy: Bryonia

The interventions were classified as Single Remedy group and Combination Remedy group. Below is the treatment group classification of the remedies.

Single Remedy Group: Hepar Sulphericum

Combined Remedy Group: Aconite+ Belladonna+ Gelsemium+ Bryonia 
DOI: 10.21522/TIJPH.2013.05.04.Art006

ISSN: $2520-3134$

\section{Treatment allocation and study drug administration}

Participants were randomly assigned to receive either single or combination remedy taken in tablet or suspension form. Study medications were administered by the principal researcher together with field assistants at the levels of $20 \mathrm{ml}$ two times daily on each visit, with six hourly intervals between administrations. The participants were given the medication for a period of 14 day.

\section{Follow-Up of study participants}

Participants were followed-up during the study and every information concerning side effects, strange outcomes or any positive remark associated with the medication were noted.

\section{Follow-Up clinical and laboratory evaluations}

After 14-days period of the study, the participants were re-examined for presence or absence of malaria parasite in their blood sample.

\section{Laboratory diagnosis of malaria}

Detailed below are the procedures used for diagnosis of malaria:

- Place one large drop of well-mixed blood in the centre of a clean glass slide

- Using the corner of a second slide, carefully spread the drop over an area of $10-12 \mathrm{~mm}$

- To determine the correct thickness of the film, place the slide on a piece of paper. Small print should just be visible through the blood.

- Dry the film at room temperature

- Prepare 1 in 10 dilution of stock Giemsa stain

- Cover the entire smear with diluted Giemsa stain for at least 10 minutes

- Wash gentle in buffer and leave slide to dry in an upright position at room temperature

- Examine under *100 objective for the presence or absence of malaria (blood parasite)

- Report as follows:

$+=1-10$ parasites per 100 thick film fields

$++=11-100$ parasites per 100 thick film fields

$+++=\quad 1-10$ parasites per 100 single thick film fields

$++++\quad$ more than 10 parasites per 100 single thick film fields

\section{Statistical analysis}

The sample size for this study was calculated and outcomes were analyzed with excel and SPSS. Results were presented in the form of tables and interpretations made accordingly.

\section{Results and discussion}

\section{Background information of respondents}

The table 4.1 below presents information on the demographic characteristic (background information) of participants.

Table 4. 1: Background information of respondents

Variable

Frequency $(\mathrm{N}=44) \quad$ Percentage

Marital status

Single

12

27.3

Married

30

68.0

Divorced

1

2.3

Widowed

1

Male

Sex of respondents

Sex of respondents

Male

Female

Highest Level of education

No formal education 
S.H.S

Tertiary

Occupational Status

Driving

Farming

Hair Dresser

Brick Layer

Mechanics

Seamstress

Students

Trader
1

5

\section{2}

15

1

1

1

3

10

11
2.3

11.4

4.6

34

2.3

2.3

2.3

6.8

22.7

25

The study enrolled a total of $44(100 \%)$ participants in the study. Of this, males were $12(27.3 \%)$ whereas females were $32(72.7 \%)$ in number. This makes the percentage inclusion of females higher than that of males. This could mean that the overall results can best be generalized for females than compared to males.

On the basis of marital statuses, most (68\%) of the participants were married perhaps few $(2.3 \%)$ were divorced and widowed each.

Levels of education among participants range from non-formal to formal-tertiary level, with majority (77.2\%) having no formal education.

Among the occupational statuses, at least all the participants were engaged in some form of employment, with majority (34\%) of them being engaged in farming activities.

\section{Overall malaria parasitemia (MPs) profile of participants}

The table 4.2 below depicts the information on the overall MPs profile of the participants enrolled in the study.

Table 4. 2. Overall malaria parasitemia (MPs) profile of participants

$\begin{array}{rrr}\text { Levels f MPs } & \text { Frequency } & \text { Percentage } \\ + & 38 & 86.46 \\ ++ & 5 & 11.46 \\ +++ & 1 & 2.20 \\ \text { Total } & \mathbf{4 4} & \mathbf{1 0 0}\end{array}$

It is observed from the table 4.2 that majority $(86.46 \%)$ of the participants enrolled in the study had + levels of MPs whilst few (2.2\%) had +++ levels of MPs. This means that though, each participant had some form of levels of MPs in their blood sample, severe form of malaria among the participant was quite minimal.

\section{MPs profile of participants on single remedy before medication}

The table 4.3 below present's information on the MPs profile of the participants who were allocated to single remedy arm or group of the study before medication was given.

Table 4. 3. MPs Profile of Participants on Single Remedy before Medication

$\begin{array}{rrr}\text { Levels f MPs } & \text { Frequency } & \text { Percentage } \\ + & 24 & 100 \\ ++ & 0 & 0 \\ +++ & 0 & 0 \\ \text { Total } & \mathbf{2 4} & \mathbf{1 0 0}\end{array}$

It is observed from the table that all $24(100 \%)$ the participants allocated to single remedy arm or group of the study were having + levels of MPs in their blood sample before administration of the remedy. This is an indication of a mild form of malaria among the participants allocated to single remedy. 
DOI: 10.21522/TIJPH.2013.05.04.Art006

ISSN: $2520-3134$

\section{MPs profile of participants on single remedy after medication}

The table 4.4 below details the information on the MPs profile of the participants who were allocated to single remedy arm or group of the study after medication was given.

Table 4.4. MPs profile of participants on single remedy after medication

$\begin{array}{rrr}\text { Levels f MPs } & \text { Frequency } & \text { Percentage } \\ - & 23 & 95.8 \\ + & 1 & 4.2 \\ ++ & 0 & 0 \\ +++ & 0 & 0 \\ \text { Total } & \mathbf{2 4} & \mathbf{1 0 0}\end{array}$

It is seen from the table that, 23 out of 24 representing $95.8 \%$ showed no presence of MPs in their blood samples after medication when blood film test was performed. Only one participant $(4.2 \%)$ still maintains with + MPs levels after being put on medication at the end of the study when blood test was performed.

This high success rate of MPs reduction as a result of homoeopathic remedy administration is a direct indication of efficacy of homoeopathic remedies for the treatment of malaria.

\section{MPs profile of participants on combination remedy before medication}

The table 4.5 below present's information on the MPs profile of the participants who were allocated to combination remedy arm or group of the study before medication was given.

Table 4.5: MPs profile of participants on combination remedy before

\section{Medication}

$\begin{array}{rrr}\text { Levels f MPs } & \text { Frequency } & \text { Percentage } \\ + & 14 & 70 \\ ++ & 5 & 25 \\ +++ & 1 & 5 \\ \text { Total } & \mathbf{2 0} & \mathbf{1 0 0}\end{array}$

It is shown from the table that various levels of MPs were present in blood samples of the participants in varying frequencies. Most $(70 \%)$ of them had + MP levels, followed by ++ $(25 \%)$ whereas few $(5 \%)$ of the +++ levels of MPs in their blood sample.

In general, the malaria condition of the participants in the combination remedy arm or group of the study was quite mild, though $5 \%$ of them had complicated form of the diseases.

\section{MPs profile of participants on ccombination remedy after medication}

The table 4.6 below details the information on the MPs profile of the participants who were allocated to homoeopathic combination remedy arm or group of the study after medication was administered.

Table 4.6. MPs profile of participants on ccombination remedy after medication

$\begin{array}{rrr}\text { Levels f MPs } & \text { Frequency } & \text { Percentage } \\ - & 19 & 95 \\ + & 1 & 5 \\ ++ & 0 & 0 \\ +++ & 0 & 0 \\ \text { Total } & \mathbf{2 0} & \mathbf{1 0 0}\end{array}$

It is observed that $95 \%$ out of $100 \%$ of the participant who were having various levels of MPs in their blood samples showed no detection or presence of MPs following an analysis of their blood after medication perhaps 5\% still retain an MPs levels of + in their blood samples after the medication when analysis was carried out.

Comparing the success rate of MPs reduction as in single remedy group to combination remedy group, no significant difference was observed between the two groups respectively (95.8\% vs. 95\%). This indicates that homoeopathic remedy in whatever formulation as single or combination has 
almost an equal rate of treating malaria. The control group showed no improvement at the end of the study. Comparing success rates of this current study involving the use of homeopathic remedies to previous studies involving conventional anti-malarial drugs like ACT and others, there exists a large evidence of homeopathic remedies being effective for malaria treatment than the conventional antimalarial drugs in which some of the success rates reported were low.

\section{Conclusion and recommendations}

This study aimed to compare the efficacy, safety and tolerability of single and combination antimalarial homoeopathic remedies for the treatment of uncomplicated malaria observed that by comparing the success rate of treatment with single remedy to combination remedy group, no significant difference was observed between the two groups respectively (95.8\% vs. 95\%). This indicates that homoeopathic remedy in whatever formulation stand an almost equal rate of treating uncomplicated malaria as documented by this study. Additional, participants on control group showed no improvement at the end of the study. They retained their initial MPs statuses. This proves a significant efficacy of homoeopathic anti-malaria remedies.

Little or no harmful effects were noted throughout the study as compared to other therapies in previous trials in which significant effects were recorded.

Based on the findings of this study, it is therefore recommended that homoeopathy remedies be advocated for nationwide usage as it has about $90 \%$ to $95 \%$ success rate for the treatment of uncomplicated malaria with no or minimal side effect.

Again, it is recommended that the research be reproduced on a larger scale involving large samples of participant in a different geographical location to ascertain the validity of the present findings.

\section{References}

[1]. Asenso-Okyere, W.K \& Dzator, J.A. (1997). Household cost of seeking malaria care: a retrospective study of two districts in Ghana. Social Science and Medicine 45(5):659-667.

[2]. Bonilla, E. \&Rodriguez, P. (1992).Tropical diseases and socio-economic development. The case of malaria in Columbia. Presentation to WHO/PAHO Interregional Conference on Malaria, 26-30April 1992, Brasillia.

[3]. Bosman, A., Mendis, K.M (2007) A major transition in malaria treatment: The adoption and deployment of artemisinin-based combination therapies. Am J Trop Med Hyg 2007, 77 (Suppl 6):193-197.

[4]. Greenwood, B.M., Bojang, K., Whitty, C.J., Targett, T.A. (2005). Malaria. Lancet 365: 1487-98.

[5]. Hyde, J.E. (2005). Drug-resistant malaria. Trends Parasitol 2005, 21:494-8. PubMed Abstract

[6]. Kleinschmidt, I., Sharp, B., Benavente, L.E., Schwabe, C., Torrez, M. (2006). Reduction in infection with Plasmodium falciparum one year after the introduction of malaria control interventions on Bioko Island, Equatorial Guinea. Am J Trop Med Hyg 74: 972-8.

[7]. Sachs, J. and Malaney, P. (2002). The Economic and Social Burden of Malaria. Nature 415, $680-685$. Macmillan Pub. Ltd.

[8]. Shepard, D.S., Ettling, M.B., Brinkman, U., Sauerborn, R. (1991).The economic cost of malaria in Africa. Tropical Medicine Parasitol 42:199-203. 